Int Arch Allergy Immunol 1993;100:383-384

\title{
Subject Index
}

\section{Acetone 251}

Adenylyl cyclase 19

Aginate 144

AIDS vaccine 93

Airway hyperresponsiveness 367

- $\quad$ obstruction 274

Allergen characterization 263

Allergens 68

Allergic inflammation 35 Allergy 60

Alveolar macrophage 28 Anaphylactic constriction 170 Antiallergic agents 190 Anti-amoebic antibodies 338 Anti-antiidiotype 121 Antibiotic 128 Anticardiolipin 293

- $\quad$ antibodies 351

Anti-DNA 293

Antigen elimination 8

Antigen-induced eosinophilia 362

Antigen-presenting cell 8

Anti-idiotype 121

Anti-PAF agent 373

Ascariasis 283

Aspirin 156

Asthma 19, 156,274,355,362

- $\quad$ model 178

Atopic dermatitis 107

Autoantibodies 293, 307

Autocrine differentiating factor 240

Autoimmunity 2, 293

Autologous serum 209

Basidiospores 263

Basophils 219

Bat 230

Beige rats 99

Binding pattern 68

Blood levels 53

Bovine serum albumin 42, 234

Bronchial asthma 151,287

Bronchoconstriction 268

Butyric acid 240

ClrsCl inhibitor $151 \mathrm{C} 3$ polymorphism $151 \mathrm{C} 3 \mathrm{~b}(\mathrm{Bb}) \mathrm{P} 151$ Calcium entry blocker 274 Capsaicin 170 Carbamazepine 378 CD23 197 CD54 121 CD8+T cells 8 Cºsgene 68 
Chemiluminescence 135

Chemotaxis 201

Chédiak-Higashi syndrome 99

Chromium chloride 333

Clinical trial 93

Clonal specificity 12

Complement activation 135, 151, 209

- components 35

Conjunctival provocation test 185

Contact dermatitis 333

Copolymer 12

Cord blood 219

Cross-reactivity 128

Cyclic AMP 19

CysCys family 201

Cystic fibrosis 144

Cytokine 378

Dactylis glomerata (Cocksfoot) 68

Daily variations 53

Degranulation 99

Delayed-type hypersensitivity 333

Dermatitis 197

Destruction 8

Dexamethasone 190

Dicarbonic acids 251

Drug allergy 378

DS-4574 268

Dysautonomia, familial 42

E6080 178

Early phase reaction 185

Early-response inhibition 367

Eicosanoids 144

ELISA (enzyme-linked immunosorbent

assay) 128, 151, 215, 338 Entamoeba histolytica 338 EoL-1 cell line 240 Eosinophil 28

Eosinophilic leukemia cells 240 Epicutaneous patch test 107 Erythrocytes 209 Escherichia coli 144 Experimental allergic encephalomyelitis

Feldl 256 Fibroblasts 219 Ficoll® 234 N-Formyl-methionyl-leucyl-phenylalani

28 Fungal allergy 263

GM-CSF 240

GP160 93

Granule, secretory 230

Granules, giant 99

Grass pollen 68

Growth hormone-releasing factor 86

Guinea pig trachea 170

Hemolysins 144 Hemolysis 209 
Heterogeneous recognition 283 Histamine 53, 144,373

release 86, 256 HIV-1 93 HLA-DQA 248 HLA-DQB 248 House dust 355 Human Fcel receptor 344

humoral immune response 68

immunodeficiency virus 107

MIP-la 201 Hypersensitivity 373

I-A region 333 ICAM-1 121 IgAl, IgA2 68 IgE 60,68,197,355

antibody repertoire 283

triggering 344 IgGl-4 68

$\operatorname{IgM} 68$

IL-3 328

IL-8 201

Immediate-type reaction 251

Immunoblastic lymphadenopathy 378

Immunoblot 60

Immunoblotting 263

Immunogenicity 128

Immunohistochemistry 107

Immunoregulation 2, 197

Inflammatory mediators 144

Inositol phosphates 19

Intercellular adhesion molecule-1 121

Interferon 197

Interleukin-1 164

Interleukin-3 215

Interleukin-4 197

Iris antigen 314

Keratinocytes 328

Lactalbumin 42

Late phase airway inflammation 362

- $\quad$ - reaction 185

Late-response inhibition 367

Lazaroids (2-methylchroman) 362

L-Leucine methyl ester (Leu-OMe) 56

Leukocyte adhesion 121

383

Leukotriene C4 178 Leukotrienes 268 Lipopolysaccharide 47 Liposomes 115 5-Lipoxygenase inhibitor 178 Loratadine 185 Lung fragments 178 Lymphocytes 19, 156 Lymphoma 215

Macrophage elimination 115

Mast cel·1(s) 56, 86, 99, 219, 230, 319, 328

- $\quad$ - activation 344

Mepyramine 190

Methotrexate 287

Mixed connective tissue disease 351 Mononuclear cell 28 Mouse 333

Muscarinic receptors 19 Mycobacterium tuberculosis 12

$\Lambda^{1} / 8 /$ proteinofHIV 107 Neisseria meningitidis 135 Neutral endopeptidase 170 Neutrophil 28 
- $\quad$ leukocytes 135

New cytokine superfamily 201 Nickel allergy 248 Nycodenz ${ }^{\circledR} 234$

Occupational allergies 251 Ovalbumin 170

Parthenium hysterophorus 79 Passive cutaneous anaphylaxis 56 Percoll ${ }^{2} 234$

Phosphoinositide metabolism 19 Phosphoramidon 170

Plasmodium falciparum 12 Platelet-activating factor 373 Pneumonitis 287 Polymorphonuclear leukocytes 19 PolymyxinB 47

Primate airway inflammation 362 Progenitors 219

Progressive systemic sclerosis 307 Pseudomonas aeruginosa 144 Psilocybe cubensis 263

Pulmonary hypertension 351 Purification media 234

Radioligand binding 19 Ragweed 79

RBL-2H3 transfectants 344 Repirinast(MY-5116) 367 Respiratory resistance 164 Retinoic acid $328 \mathrm{Rev}$ protein of HIV 107 Rhesus monkey 178 Rheumatoid arthritis, juvenile 314

- $\quad$ factor 293

RNP 293

RWJ 22108274

S antigen 314 SC5b-9 151 Scleroderma 307 Scroll-containing granules 230 Serum albumin, human 42

- $\quad$ bactericidal activity 135

sICAM-1 121

Silica 164 Skin 107

prick test 251, 256

reactivity 190 SM10661 373

Sodium fluoride 234 SSA 293 SSB 293

Staphylococcus aureus 144 Stem cell factor 328 Streptococci 12 Streptozotocin 115 Sucrose 234

Superantigen 12 Superoxide anion 28 Suppression 8 Suppressor cells 2

Systemic lupus erythematosus 107, 215, 293,351

T cells 333

- lymphocytes 35

Tat protein of HIV 107

Therapy 93

Thin-layer immunoassay 338

$\gamma \delta$ T lymphocytes 12

Tolerance 2

2,4-Toluene diisocyanate 190

Transformation 319

Tumor toxicity 47

Tumoricidal activity 47

U931 cell 28 UCD200 307 Ultrastructure 219 Urticaria 107 Uveitis 314

Vasoactive intestinal pep tide $28 \mathrm{~V} \gamma 9 \mathrm{~T}$ cell receptor 12 Virus 319

Zymosan 209

384

Subject Index 